\title{
Robopal: Modeling Role Transitions in Human-Robot Interaction
}

\author{
Dylan F. Glas, Takahiro Miyashita, Hiroshi Ishiguro, and Norihiro Hagita \\ ATR Intelligent Robotics and Communication Laboratories \\ 2-2-2 Hikaridai, Seika-cho, Souraku-gun, Kyoto, 619-0288, Japan \\ $\{$ dylan, miyasita, ishiguro, hagita\}eatr.jp
}

\begin{abstract}
We have developed a new communication robot, Robopal, which is an indoor/outdoor robot for use in humanrobot interaction research in the context of daily life. Robopal's intended applications involve leading and/or following a human to a destination. Preliminary experiments have been conducted to study nonverbal cues associated with leading and following behavior, and it has been observed that some behaviors, such as glancing towards the leader or follower, appear to be roledependent. A system for representing these behaviors with a state transition model is described, based on four kinds of interaction roles: directive, responsive, collaborative, and independent. It is proposed that behavior modeling can be simplified by using this system to represent changes in the roles the robot and human play in an interaction, and by associating appropriate behaviors to each role.
\end{abstract}

\section{INTRODUCTION}

We like to think of ourselves as the masters, and of machines as our slaves. This is not necessarily the case.

Robots have begun moving off of factory floors, out of research labs, and into our homes and lives. Consumer robots like Roomba [1] are moving beyond the status of mere "bleeding edge" novelties for technophiles and hobbyists and beginning to earn acceptance in society. These robots are still far from anything we could consider social peers, but when the time comes for robots to truly emerge and become a part of our society, what roles will they play?

Although a butler-like role is still the image held by many [2], laboratory studies and field trials are moving in a different direction, putting robots into roles such as tour guides [3], receptionists [4], and guides for the blind [5]. These are positions of authority, and when robots filling these roles tell us what to do, we will naturally be expected to obey.

If robots in society are to lead us as well as follow, then our social relationships with them will not be simple. Clear communication of roles and expectations will be essential for humans and robots to build trust and work together smoothly.

At the same time, current advances in the field of HumanRobot Interaction (HRI) are enabling our interactions with robots to become less mechanical and more social. As robots begin to engage our emotions and not just our intellects, primitive push-button interfaces and warning buzzers are giving way to more subtle channels of implicit and nonverbal communication which make direct use of our natural facilities for social interaction [6]. Voice tone, eye contact, facial expressions, subtle body movements, relative positioning, and other forms of implicit communication are fundamental to the way humans communicate in everyday life, and for

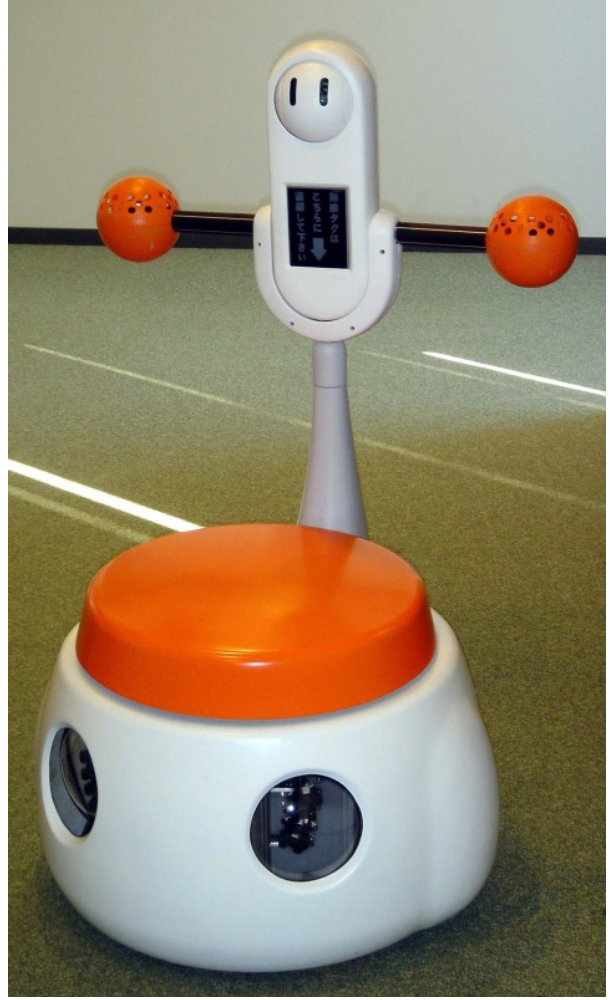

Fig. 1. Photo of Robopal

robots to participate in our society, they will need to begin communicating in those ways too.

This paper presents a new approach to modeling and simplifying the development of robot behaviors for interaction with humans in everyday situations, based on the changing roles of the robot and human. In this role-transition model, sets of behaviors are mapped to leading, following, and other roles within an interaction, so that as the roles change, the behaviors for each role can be treated as a group rather than managed individually.

We have developed a new communication robot, Robopal (Fig. 1), for HRI research in the context of everyday life as a part of the Network Robot project [7]. Section II of this paper describes Robopal's design and applications. The roletransition model presented here is a part of ongoing research into leading and following behaviors using Robopal.

Section III motivates the role-transition model and discusses its implications for robot behavior and interaction design. Section IV describes preliminary experiments which 
have been conducted regarding human-human leading and following behavior and discusses their implications regarding role transitions and human-robot interaction. Finally, Section $\mathrm{V}$ presents conclusions and areas for future work.

\section{ROBOPAL}

The discussion and observations in this paper center around Robopal, a new type of communication robot that we have developed for HRI research. Robopal is designed to operate in the domain of everyday life, acting as a guide or helping people with daily errands in real-world environments. For these tasks, Robopal's unique physical form gives it practical advantages over many existing robot designs.

\section{A. Concept}

Robots of many forms have been developed as platforms for HRI research. Although many have basic humanoid features, such as a head and arms, a wide range of body types can be seen. These include biped or wheeled humanoids [8], [9]; dinosaurs [10], dogs, and other animals [11]; and abstract forms that resemble nothing in nature, such as Cero [12] and Muu [13].

The challenge with Robopal was to design a robot optimized for helping a human user with daily errands. Such a robot needs to be able to lead or follow a person through indoor or outdoor environments and carry baggage for its user. Most importantly, Robopal is a communication robot, so it needs to be able to communicate using sound and gestures.

\section{B. Design}

Robopal's physical form was designed with these basic requirements in mind. Its form is distinctly non-humanoid, although it has a simple, abstract face to provide a focus for communication. Its base is a platform capable of carrying baggage, and it has two handles which can provide physical support for an elderly user walking behind the robot. Technical specifications for the robot are summarized in Table I.

Robopal has a sturdy wheelbase designed for indoor or outdoor use, equipped with all-terrain $15 \mathrm{~cm}$ solid rubber tires. Its wheelbase uses a two-wheel-steering configuration with powered front and rear wheels, shown in Fig. 2, which was chosen for reliable traction on uneven ground and for improved maneuverability over a differential-steering system. This wheel configuration also enables Robopal to move holonomically, i.e. orient itself in a direction independent of the direction of motion, which is one possible way of conveying a distinction between direction of attention and direction of motion.

Robopal can move its head with three degrees of freedom: its face can rotate up and down (pitch) or side to side (yaw), and its neck can also swivel left and right (yaw), as illustrated in Fig. 3. These motions are directly driven by three servomotors mounted in the robot's head. Note that the simplicity of the facial features is intentional, in order to
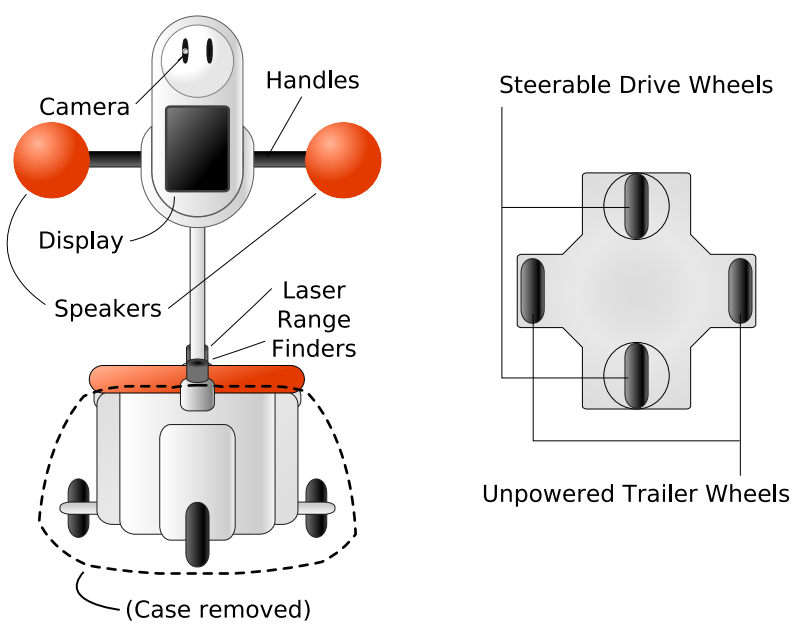

Fig. 2. Robopal layout and wheel configuration.

enable gestural cues from the robot to be easily understood even by elderly users with poor eyesight.

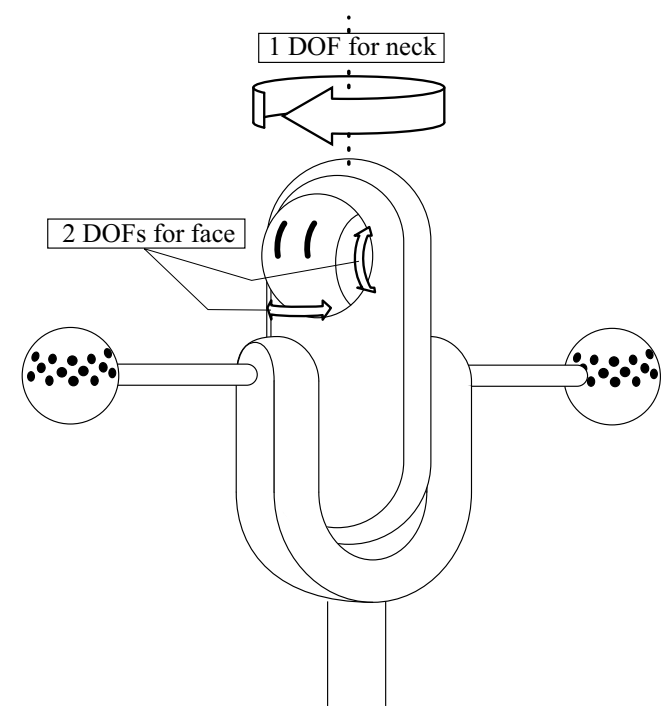

Fig. 3. Degrees of freedom in Robopal's head and neck

Robopal uses an internal map to localize itself relative to objects in the environment. Dead reckoning estimates of its current position are derived from the shaft encoders in the drive wheels. These estimates are then refined, using data from laser range finders mounted on the front and rear of the base. A greedy algorithm is used to correct the position estimates by iteratively applying displacements which locally minimize the mean-squared error between the sensor data and the nearest features on the internal map.

With this map-matching algorithm, and using a system of waypoints designated on the internal map, we have demonstrated that Robopal can consistently navigate safely along a 63-meter hallway path with five $90^{\circ}$ turns. 
TABLE I

TECHNICAL SPECIFICATIONS FOR RoBOPAL

\begin{tabular}{|l|l|l|}
\hline \multirow{3}{*}{ Body Dimensions } & Height & $1200 \mathrm{~mm}$ \\
& Width & $580 \mathrm{~mm}$ \\
& Length & $620 \mathrm{~mm}$ \\
& Weight & Approx. 30 kg \\
\hline \multirow{2}{*}{ Performance Limits } & Max. Driving Speed & $1.0 \mathrm{~m} / \mathrm{sec}$ \\
& Battery Life & Approx. 3 hours \\
\hline \multirow{5}{*}{ Hardware Components } & Onboard PC & Sony VAIO VGN-U50 (Celeron 900 MHz, 256 MB RAM, 20GB HD) \\
& Distance Sensors (x2) & Hokuyo URG-04LX Scanning Laser Range Finders \\
& Camera & Akizuki DX-670 VGA Webcam with microphone \\
& Servomotors (x3) & Sanwa ERG-VB High Speed / High Torque Servos \\
& Motors (x4) & Maxon RE-36 70-Watt DC Motors \\
& Motor Controllers (x2) & VSTONE VS-R230A Motor Controller Boards \\
& Batteries (x2) & Yuasa STURD II 12V / 28Ah car batteries \\
\hline
\end{tabular}

\section{Applications}

Robopal was designed for two general categories of communicative applications. The first category, assistive applications, includes tasks in which the human determines the goals, and the robot uses its abilities to help the human achieve those goals. The second category, guiding applications, includes tasks where the robot is primarily in control, using its knowledge and abilities to guide the human. These roles are not necessarily fixed at all times, but they reflect the overall operating relationship between the robot and the human. Some examples of these applications follow.

1) Assistive Applications: Shopping assistance is one example of an assistive application for which Robopal is well-suited. In such an application, a number of robots would be located at a shopping center or large store. They would greet customers as they enter, and offer to accompany them through the store. All decisions as to what products to look for, when to give up searching, and where to go next, would be determined by the human. The robot would provide support by helping to find products, providing information relevant to purchases, carrying heavy items for the shopper, and making the shopping trip a more pleasant and entertaining experience by chatting with the human.

Another assistive application would be following a user throughout the day and helping with daily errands in general, which is one of the primary applications for which Robopal has been designed. This is a much more complex problem than a store-specific shopping robot, due to the variability of environmental conditions and the variety of errands that might require different behaviors. Such an application might be particularly useful for elderly users. The robot could accompany the user on a trip into town, helping to carry things like dry cleaning and letters or packages to be sent, and carrying groceries on the way back. En route, the robot could also help the user by performing monitoring tasks, for example keeping track of the user's pace for an exercise log, or making an emergency phone call if the user collapses. Socially, the robot could also talk to the user to provide companionship. At times when the human needs help for navigation, the robot could take a leading role, but in general the robot would follow the human.

2) Guiding Applications: In guiding applications, the robot primarily plays a leading role, and the human follows the robot's directions or guidance.

One example of a guiding application is giving tours of a museum. Tour-guide applications like this have often been used as a context for studying and developing socially interactive robots [3], [14], [15]. Since the robot has expert knowledge of the area, the robot is usually in a leading role, and the humans follow it around.

A hotel is another possible setting for a guiding robot application. Consider a scenario in which a couple arrives at an upscale hotel. They check in, and are greeted by the robot. Rather than calling a human porter to carry their luggage, they simply place their bags on the robot's luggage rack, and the robot politely shows them the way to their room. On the way, the robot might explain about additional hotel services which are available, or give the couple information on local tourist attractions. In this scenario, highly polished communication skills would be quite important, as the robot would be representing the hotel and its reputation.

A similar application would be useful at an airport, a chaotic environment where counter and numbers can be confusing, and many people are carrying heavy luggage. A robot could greet people at the door, offering to help carry their bags and guide them to the proper ticket counter. Here, again, the robot's location-specific knowledge naturally places the robot in a leading role.

\section{Research Focus}

In all of these scenarios, Robopal needs to lead or follow a person to a destination. For this reason, current research with Robopal is focused on understanding and reproducing the nonverbal communication cues used by people as they lead and follow one another. Since the roles of leader and follower (and other roles, as the next section explains) will often change over the course of an interaction, it is also necessary to understand and model the ways in which these role transitions occur. 


\section{ROLE-TRANSITION MODEL}

At the heart of much of today's HRI research is the goal of creating robots that can interact with people in a comfortable and natural way. Implicit behavior, which includes both nonverbal communication and also implicit verbal cues such as changes in voice quality [16], plays an essential role in this research. Spoken language interfaces are often technically infeasible and are inappropriate for some applications [17]. However, gesture, gaze, and proximity convey a wealth of information on both conscious and subconscious levels, and many current research efforts are exploring ways to utilize these channels effectively in human-robot communication [18] [19].

Such studies are essential to understanding and replicating implicit communicative behaviors, yet they tend to be taskspecific, bottom-up studies. Actual implementation of these behaviors in a real-world robotic application remains challenging and complex. This is particularly true when a robot needs to perform many different functions, since a gesture or signal in one context may not have the same meaning in another context. Thus, complex sets of behaviors must be designed for each context in which the robot must operate, each with its own sequences of gestures and interaction rules.

\section{A. Changing Roles in Human-Robot Collaboration}

Consider the case of a vending machine. A human user inserts money and pushes a button to give a command to the machine, which then responds in a predictable way. At all times the human is taking the initiative and the machine is reacting to the human's actions.

Social interactions, however, are seldom so simple. When people work together on complex tasks in a real-world setting, the roles of leader and follower alternate based on context. This back-and-forth exchange is a fundamental part of social collaboration, emerging from the fact that people depend on each other for their different strengths. Social robots will also need to participate in this alternation of roles, particularly since robots differ so greatly from humans in terms of their strengths and weaknesses.

For this discussion, let us designate four types of interaction modalities, or roles: "directive", "responsive", "collaborative", and "independent", and define them as follows:

1) Directive and Responsive: In a directive-responsive interaction, the directive participant is the one initiating actions, and the responsive participant is the one responding to those actions. In all of the applications mentioned above, Robopal is required to physically lead or follow a human. While physical leading and following are very clear examples of directive and responsive behavior, these terms are intended to be more general and can refer to any interaction of a directive nature, such as one participant directing a conversation by proactively asking questions. In many cases the directive participant is the one with more knowledge of the shared goal.

It should be noted that these designations refer to the nature of the immediate interaction, and they are independent of the actual social relationship of the participants. Thus a robot servant might ask whether its owner would prefer coffee or tea. In terms of social standing, the human is the leader and the robot follows the orders of the human, but in terms of this specific interaction, the robot is taking the directive role.

2) Collaborative and Independent: In a collaborative or independent interaction, neither party is directing the other. As the names suggest, collaborative indicates a situation where the human and robot are engaged in a task together, and independent implies that they are pursuing separate tasks entirely.

These two roles are somewhat more difficult to define than in the directive-responsive case; however, in a collaborative task, the human and the robot are working together, meaning that they are constantly paying attention to each other as well as to the task at hand. Independent tasks, by comparison, can be executed without the human or the robot paying attention to each other.

\section{B. Implications for Robot Design}

Designing natural behaviors for a robot involves defining several types of behaviors. Some may be long, non-repeating sequences of actions, some may be shorter actions repeated at intervals or continuously, and others may be rules for reacting to human actions or environmental changes. As the behaviors become more realistic and well-developed, and as the number of functions the robot can perform increases, the rules defining these behaviors will become quite complex. The purpose of defining these roles is to create a conceptual framework which can help organize these behaviors, making them more structured and manageable.

Two steps are required to take advantage of these role definitions to simplify behavior design. First, role-specific behavior patterns need to be identified and implemented for each task the robot will perform. Then, rules must be formulated to direct the transitions between roles.

\section{Modeling Role Transitions}

Just as humans must perform different sets of roles for different jobs, robots designed for different functions in society will also perform different patterns of role transitions. The rules for these transitions will depend on the particular application of the robot, and two examples of common types of transitions will be given here.

1) Example 1: A Tour-Guide Robot: For the first example, let us examine one possible sequence of actions that might be taken by a guiding application, in this case a tour-guide robot.

Consider the scenario of a museum guide robot looking for a human to show around the museum. The robot is operating in an independent state, either waiting by the entrance or wandering around the museum. (a) When it locates a human, it transitions to a directive mode, and it approaches the human to offer its services. (b) If the human accepts the offer, the robot tries to determine what part of the museum the human would enjoy visiting, perhaps through a collaborative process using an onboard graphical interface. (c) Once a 


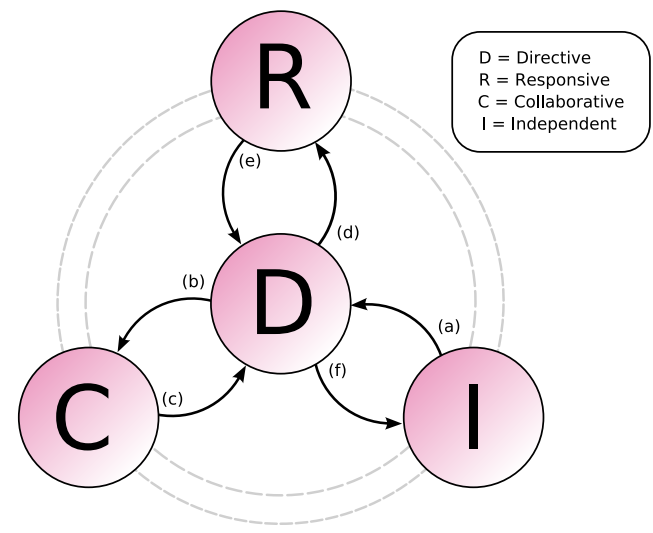

Fig. 4. Role Transitions for Tour-Guide Robot Example

TABLE II

TRANSITION RULES FOR TOUR-GUIDE RoBOT EXAMPLE

\begin{tabular}{|c|c|l|}
\hline Transition & State Change & Reason for Transition \\
\hline \hline (a) & $\mathrm{I} \rightarrow \mathrm{D}$ & Human sighted \\
\hline (b) & $\mathrm{D} \rightarrow \mathrm{C}$ & Robot needs to determine destination \\
\hline (c) & $\mathrm{C} \rightarrow \mathrm{D}$ & Destination chosen, robot begins tour \\
\hline (d) & $\mathrm{D} \rightarrow \mathrm{R}$ & Human takes detour \\
\hline (e) & $\mathrm{R} \rightarrow \mathrm{D}$ & Detour finished, robot resumes tour \\
\hline (f) & $\mathrm{D} \rightarrow \mathrm{I}$ & Tour complete \\
\hline
\end{tabular}

destination has been chosen, the robot again takes a directive role, guiding the human through the museum. (d) At times along the tour, the human may choose to spend longer at one exhibit or explore an area outside of the path of the tour. In this case, the robot might be programmed to operate in a responsive mode, and stay with the user during the detour. (e) After the detour is over, the robot returns to its directive tour-guide behavior. (f) At the end of the tour, the robot returns to its independent neutral state, looking for another human to guide.

These transitions are summarized in Table II. The purpose of the tour-guide robot is to provide guidance and information, so it naturally operates primarily in a directive mode. Fig. 4 illustrates these state transitions. This time the directive role is depicted in the center because it is the robot's main operating state.

2) Example 2: A Shopping Robot: For another example of role transitions, consider a robot-assisted shopping scenario, another active field in recent research [20] [21].

The robot begins in a waiting state near the store entrance. (a) When a customer enters, the robot comes out of its waiting state to greet the customer and begins following the customer through the store, in a responsive role. In this case, the human is familiar with the store and knows where to go, thus taking the directive role. (b) At some point the customer does not know where to find a product, and asks the robot. The roles are then reversed, as the robot uses its knowledge of the store to guide the customer to the most likely location. (c) Suppose then that the product is not there. The robot and

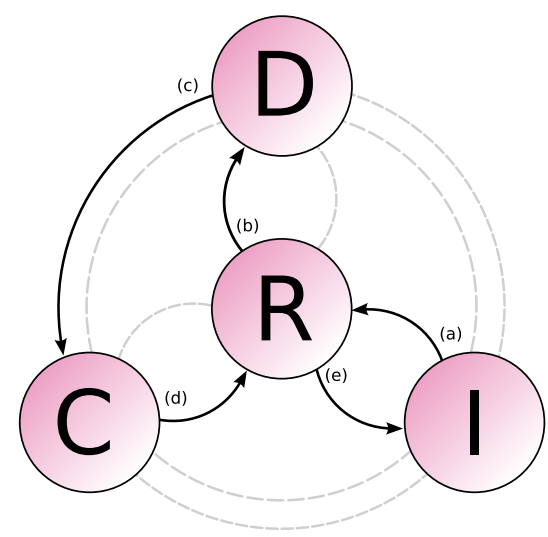

Fig. 5. Role Transitions for Shopping Robot Example

TABLE III

Transition RULES FOR SHOPPING RoBOT EXAMPLE

\begin{tabular}{|c|c|l|}
\hline Transition & State Change & Reason for Transition \\
\hline \hline (a) & $\mathrm{I} \rightarrow \mathrm{R}$ & Customer enters store \\
\hline (b) & $\mathrm{R} \rightarrow \mathrm{D}$ & Customer requests help \\
\hline (c) & $\mathrm{D} \rightarrow \mathrm{C}$ & Product not found \\
\hline (d) & $\mathrm{C} \rightarrow \mathrm{R}$ & Problem resolved \\
\hline (e) & $\mathrm{R} \rightarrow \mathrm{I}$ & Customer finishes shopping \\
\hline
\end{tabular}

human may then work in a collaborative mode to explore their options. The customer can easily search the surrounding area to see if the product was misplaced, whereas the robot can access the store database to determine whether the item might be available at another location. (d) Once the customer decides the situation is resolved, the robot returns to its responsive role, following the customer and offering help until the customer leaves. (e) Once the customer has left, the robot returns to an independent waiting state near the entrance.

These transitions are summarized in Table III. It is clear that the role-transition model for the shopping robot differs greatly from that of the tour-guide robot. These two robots have fundamentally different relationships with the humans with whom they interact. In this assistive application, the robot's primary role is responsive in nature, so the robot returns to a responsive state by default, as represented in Fig. 5.

\section{Role-Dependent Behaviors}

In each of the above examples, the robot operates in all four roles. To make effective use of the role-transition model, appropriate behaviors must be identified that correspond with each of the four roles. Although these behaviors should be determined through research and experimentation, firstpass assumptions can be made about what general types of behaviors are likely to be effective. Several likely candidates for role-specific behaviors are presented in this section, and the results of a preliminary experiment to empirically identify role-specific behaviors will be presented in section IV. 
1) Directive Behaviors: In a directive mode, the robot should convey an implicit message that it is alert and in charge. The impression it conveys should make the human feel comfortable trusting the robot and following its lead.

If a robot is in a directive state and does not clearly convey that fact, the human may not pay attention to the robot's instructions or guidance. Even if it does convey its intentions, the robot needs to make the user feel safe and comfortable trusting it, or the user may choose not follow the robot's directions at all.

2) Responsive Behaviors: A robot in a responsive mode should appear attentive, giving feedback to the human that indicates that it is listening and ready to accept commands. This could involve steady or periodic eye contact, open body language, and motions such as nodding or inclining the head to give the appearance of listening. Feedback is also important to confirm that the robot understands what the user has said.

If the robot does not appear attentive, the user may become frustrated when trying to give the robot instructions or convey information.

3) Collaborative Behaviors: In a collaborative interaction, the robot's attention should appear divided between the human partner and the task at hand. This might be communicated by directing the robot's gaze back and forth between the human and the physical focus of the task (if there is one), or through body positioning, facing partly towards the human and partly towards the focus of the task. Many variations of these techniques are conceivable, such as facing the body in one direction and the head or eyes in another, to convey divided attention. Collaborative tasks with no physical focus would require different body language, such as perhaps looking up and to the side while doing time-consuming calculations or accessing online information about the task.

A robot operating in a collaborative role that appears too focused on the task may make the user think it is operating in an independent role, thus discouraging the user from trying to work together with it.

4) Independent Behaviors: In an independent mode, the robot should indicate that it is focused on its current task. It should also indicate how busy it is, so the human knows whether to interrupt or not.

A robot operating in an independent mode but which seems to be in a responsive mode may cause confusion by making the user think the robot is waiting for information from the human. Likewise, a robot should not convey through body language that it is operating in an independent mode if it is actually waiting for the human to take action.

\section{EXPERIMENT}

We conducted a preliminary experiment to study implicit human behavior while leading or following another human. Intuition and personal experience suggest that some of the motions involved in walking, such as head and eye motion, or relative walking position, are role-dependent. The goals of this experiment were, first, to gather data in support of or in

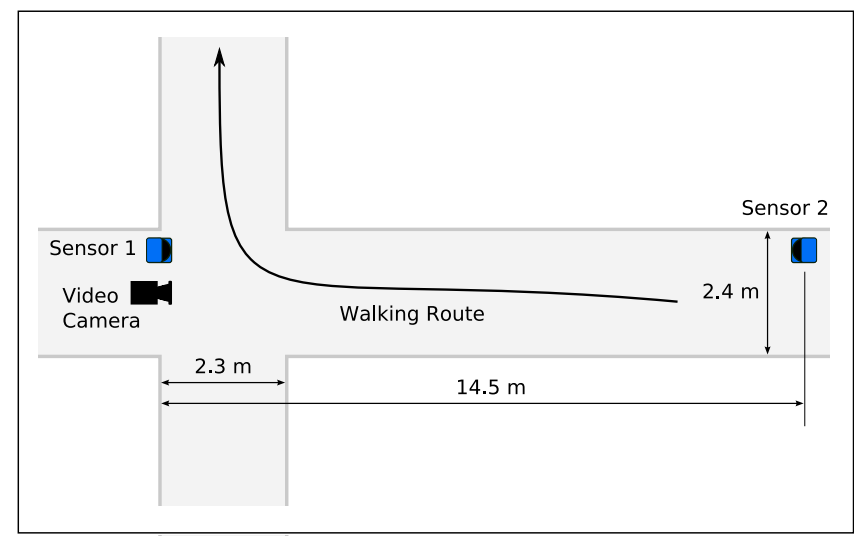

Fig. 6. Experiment Setup

conflict with this assertion, and, second, to identify which elements of nonverbal communication appear to be roledependent and might be good candidates for implementation in a robot.

\section{A. Experiment Design}

1) Setup: This experiment was conducted at ATR, in a length of hallway ending in a four-way intersection. In order to accurately track the positions of the subjects along the hallway, two SICK LMS-200 infrared laser range sensors were used, one at each end of the hallway, as shown in Fig. 6 . The sensor at the intersection was positioned to enable the motion of the subjects to be measured for several meters in either direction after turning the corner. A video camera was also positioned at the end of the hallway.

In addition to video and position data, the eye and head movements of each of the subjects were measured using wearable EMR-8B Eyemark Recorder eye trackers (NAC Image Technology, Inc.). The video output from the Eyemark Recorder shows the point of a subject's visual focus superimposed on a video image from a head-mounted camera. From this video image, the directions of the subjects' head motions and eye movements were determined.

2) Procedure: Seven subjects participated in the experiment. Due to the amount of time required to calibrate the eye trackers, the most efficient procedure was for each subject to participate in two consecutive sets of trials with different partners. Thus six pairs of subjects were recorded.

Each trial consisted of one subject leading another down the hallway and turning left or right, and trials were executed in pairs. In the first trial of each pair, the leader was instructed to choose a direction (right or left) and then to lead the other subject down the hall and around the corner in that direction. In the second trial of each pair, the same leader was instructed to lead the other subject to the same destination. The follower was also informed that the second trial would be in the same direction. The reason for conducting pairs of trials in this way was to distinguish whether the observed behaviors are dependent upon whether the follower has $a$ priori knowledge of the destination. In total, eight trials 


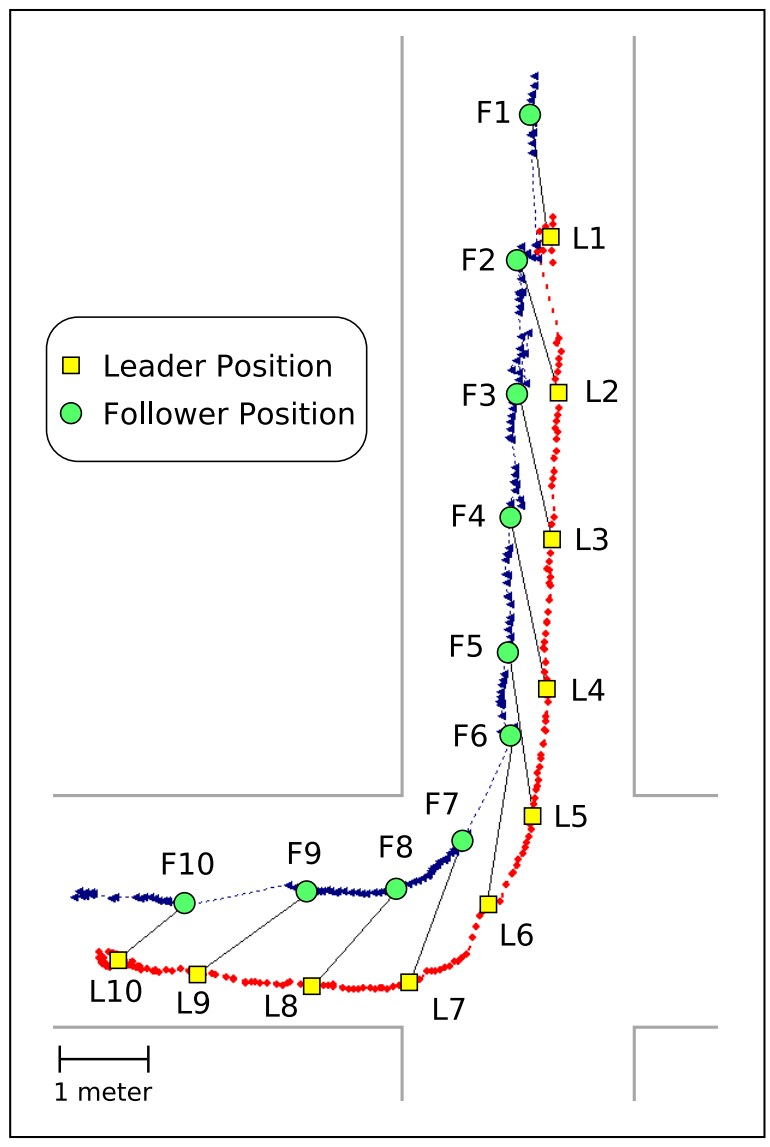

Fig. 7. Leader and Follower Positions Measured at 1-Second Intervals

were conducted with each pair of subjects, with each partner designated as leader for four of those trials.

\section{B. Results}

Position data from the range sensors was recorded for each trial, from which the positions of the subjects were then determined. Fig. 7 shows a typical path taken by a pair of subjects. When the subjects occluded one another, particularly through areas covered by only one of the two range sensors, it was impossible to determine their distance and identity, so there are many gaps in the position data for most trials.

In general, two types of leading-following behavior were observed. Some pairs of subjects tended to walk nearly sideby-side, with the leader slightly ahead of the follower. Other pairs walked with the leader almost directly ahead of the follower. In the single-file cases, there appeared to be little or no interaction between the subjects while walking.

In the side-by-side cases, there was a tendency for the leader to glance back periodically, presumably to confirm that the follower was still there. These glances usually involved head movements on the order of 30-70 degrees, and no eye contact was made. In fact, in nearly all cases the follower's face was not visible on the video image, although the leader's visual focus point was visible. This indicates that the leader may have been identifying the follower through peripheral vision.

The follower, on the other hand, tended to look directly at the leader's face without a large amount of head movement. This behavior was most often observed in two situations: (1) immediately after the leader glanced back at the follower, and (2) when the leader began to turn around the corner.

In many cases, it seemed clear which of the subjects initiated the single-file behavior. In some trials, the leader looked back repeatedly to try to find the follower, who was walking some distance behind. In others, the leader looked directly ahead without taking any notice of the follower, even though the follower glanced at the leader often.

No official questionnaire was given to the participants, but one subject's comments are worth noting. This subject walked with two other subjects, one of whom led by walking side-by-side with the subject, glancing back periodically, and one of whom led in a single-file way, without glancing back. The subject's impression was that the side-by-side leading conveyed an impression of friendliness, whereas the singlefile leading without looking back seemed somewhat rude.

This comment underscores the need to be aware that some human behaviors, although authentic, may be undesirable. It is important to keep in mind that the goal of developing behaviors for social robots is more than simply emulating human behavior - it is emulating effective human behavior. Careful thought must be given to what kinds of human behaviors are reproduced, and it is even conceivable that new, robot-specific sets of behaviors will emerge - behaviors that may not even be physically possible for humans, but which effectively enable good communication and harmonious coexistence between humans and robots.

\section{Discussion}

The results of this study provide indications of critical patterns in leading and following behaviors. The overall glancing behaviors of the leader and follower appear to be fairly consistent between subjects, and there seems to be a consistent difference between the behaviors of the directive partner and responsive partner for this type of interaction. This clear distinction between role-specific behaviors seems to indicate that the proposed role-transition model could be applied to interactions involving leading and following.

Behaviors modeled after those observed in this experiment are now being implemented in Robopal, and further experiments will investigate the importance of these behaviors and the effectiveness of different implementations of those behaviors using the robot. Once effective behaviors have been developed for each of the proposed roles, it will be possible to begin studying the effectiveness of their use as part of an application in a real-world setting.

\section{CONCLUSIONS AND FUTURE WORK}

\section{A. Conclusions}

This paper has presented a new approach for modeling human-robot interactions. In a real-life application, the immediate interactive relationship between a robot and human 
will change frequently, with the human and the robot dynamically moving between directive, reactive, collaborative, or independent roles depending on context. We have proposed that associating sets of behaviors with these roles might become a basis for simplifying the design of robot behavior rules for complex tasks.

A new robot, Robopal, has been designed to work with and assist people in everyday life. Applications for a robot like Robopal are likely to involve many of these role transitions, so a preliminary experiment was conducted to study the behaviors of humans in leading and following roles in a guiding situation. Results of this experiment suggest that there are observable distinctions between the behaviors associated with a leading (directive) role and the behaviors associated with a following (responsive) role. These results imply that the role-transition model may be a useful framework for modeling these behaviors in a social robot.

\section{B. Future Work}

Several aspects of this model remain to be investigated. For example, in this experiment, only directive and responsive roles were considered. Behaviors specific to collaborative and independent roles also need to be identified. In addition, the observation of human interactions in a wider variety of task contexts may reveal additional role-dependent behaviors. For example, some aspects of body language used in face-to-face tasks may differ from that in the leading and following task studied here.

Beyond the question of identifying the role-dependent behaviors themselves, this model also prompts the fascinating question of how role transitions are signaled. What are the implicit communicative cues that humans use to negotiate a role transition? Identifying these behaviors will be of great value to the development of socially-situated robots.

We believe that the model presented in this paper is a powerful concept for the organization and categorization of behaviors. To realize its full potential, a larger set of interactions should be studied, to create a more comprehensive list of behaviors associated with each of the roles. As these behaviors are identified, the role-transition model presented here will provide a useful starting point for further organization of behaviors into more fine-tuned categories and subcategories.

Finally, the high-level goal for this research is to produce basic applications using Robopal and test them in real-world environments such as shopping centers or airports to further explore and overcome the practical and theoretical challenges of developing robots as participants in society.

\section{ACKNOWLEDGMENTS}

This research was supported by the Ministry of Internal Affairs and Communications.

\section{REFERENCES}

[1] iRobot Corporation, Burlington, MA, USA. http://www.irobot.com

[2] K. Dautenhahn, S. Woods, C. Kaouri, M. Walters, K. Koay, and I. Werry, "What is a robot companion - friend, assistant or butler?" In Proceedings IEEE/RSJ International Conference on Intelligent Robots and Systems, pp. 1488-1493. Edmonton, Canada, 2005.

[3] S. Thrun, M. Bennewitz, W. Burgard, A.B. Cremers, F. Dellaert, D. Fox, D. Haehnel, C. Rosenberg, N. Roy, J. Schulte, and D. Schulz, "MINERVA: A second generation mobile tour-guide robot", Proceedings of the IEEE International Conference on Robotics and Automation (ICRA'99), 1999.

[4] R. Gockley, A. Bruce J. Forlizzi, M. Michalowski, A. Mundell, S. Rosenthal, B. Sellner, R. Simmons, K. Snipes, A.C. Schultz and J. Wang, "Designing Robots for Long-Term Social Interaction", Proceedings of IROS 2005, Edmonton, Alberta, 2005.

[5] V. Kulyukin, C. Gharpure, J. Nicholson, "RoboCart: Toward RobotAssisted Navigation of Grocery Stores by the Visually Impaired", Proceedings of the IEEE/RSJ International Conference on Intelligent Robots and Systems (IROS 2005), Edmonton, Alberta, Canada, 2005.

[6] C. Breazeal, Designing Sociable Robots, MIT Press, 2002.

[7] T. Kanda, H. Ishiguro, and N. Hagita, "Human Robot Communication for Network Robots - insights from field experiments", Proceedings of Workshop on Network Robot System at IEEE/RSJ International Conference on Intelligent Robots and Systems, 2004.

[8] T. Kanda, H. Ishiguro, M. Imai, T. Ono, "Development and Evaluation of Interactive Humanoid Robots", Proceedings of the IEEE (Special issue on Human Interactive Robot for Psychological Enrichment), Vol.92, No.11, pp. 1839-1850, 2004

[9] K. Kaneko, F. Kanehiro, S. Kajita, H. Hirukawa, T. Kawasaki, M. Hirata, K. Akachi, and T. Isozumi, "Humanoid Robot HRP2", Proceedings of IEEE International Conference on Robotics and Automation, pp. 1083-1090, 2004.

[10] Tmsuk Co., Ltd., Kitakyushu, Japan. http://www.tmsuk.co.jp/english/

[11] T. Nakata, T. Sato, T Mori, "Producing Animal-like and Friendly Impressions on Artifacts and Analyzing their Effect on Human Behavioral Attitudes", IEEE Int. Conf. on System, Man and Cybernetics (SMC '99), 1999.

[12] K. Severinson-Eklundh, A. Green, and H. Huttenrauch, "Social and collaborative aspects of interaction with a service robot", Robotics and Autonomous Systems, Special Issue on Socially Interactive Robots, 42, 3-4, March 2003.

[13] M. Okada, S. Sakamoto and N. Suzuki, "Muu: Artificial Creatures as an Embodied Interface", Proceedings of 27th International Conference on Computer Graphics and Interactive Techniques (SIGGRAPH 2000), the Emerging Technologies: Point of Departure, p.91, 2000.

[14] W. Burgard, , A. B. Cremers, , D. Fox, , D. Hahnel, , G. Lake-meyer, , D. Schulz, , W. Steiner, and S. Thrun, Experiences with an interactive museum tour-guide robot, Artificial Intelligence 114(1-2): 3-55, 1999.

[15] M. Shiomi, T. Kanda, H. Ishiguro, N. Hagita, "Interactive Humanoid Robots for a Science Museum", ACM 1st Annual Conference on Human-Robot Interaction (HRI2006), pp. 305-312, Salt Lake City, Utah, USA, March 2006

[16] C.T. Ishi, H. Ishiguro, and N. Hagita, "Using Prosodic and Voice Quality Features for Paralinguistic Information Extraction", $C D-R O M$ Proceedings of The 3rd International Conference on Speech Prosody (SP2006), 2006.

[17] V. Kulyukin, "On Natural Language Dialogue with Assistive Robots", Proceedings of the 2006 ACM Conference on Human-Robot Interaction (HRI 2006), Salt Lake City, Utah, 2006.

[18] C. Breazeal, C. D. Kidd, A. L. Thomaz, G. Hoffman, and M. Berlin, "Effects of nonverbal communication on efficiency and robustness in human-robot teamwork", Proceedings of IROS, Barcelona, 2005.

[19] A. Brooks and R. Arkin, Behavioral overlays for non-verbal communication expression on a humanoid robot, Autonomous Robots, Online Publication, September, 2006.

[20] NTT Communications, news release (in Japanese), http://www.ntt.com/release/2006NEWS/0002/0202.html, 2006.

[21] G. Garcia, unpublished project, "Battery Operated Smart Servant", Intelligent Machines Design Laboratory, University of Florida, http://mil.ufl.edu/5666/ 